
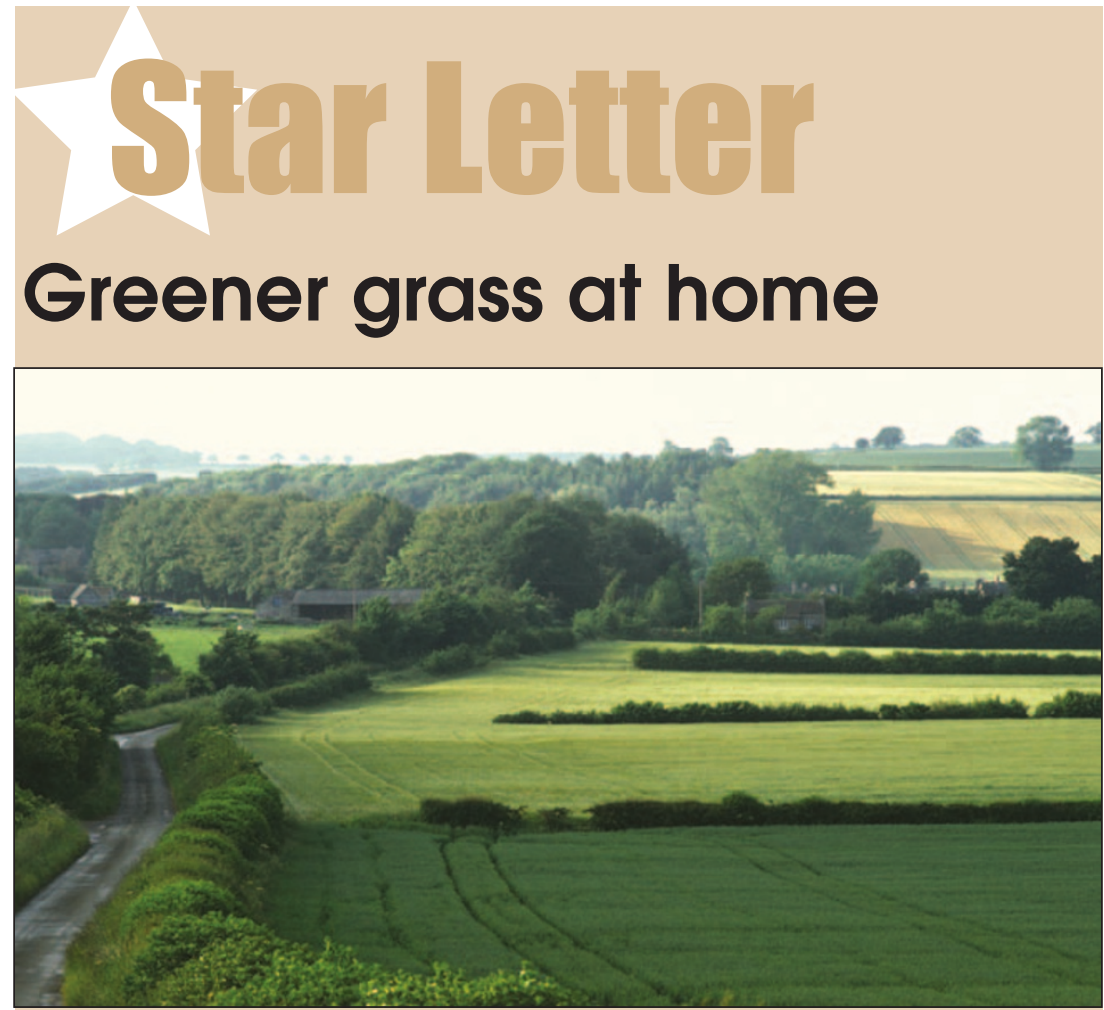

I had to write to compliment you on the introduction of Vital, which has every sign of being a useful and enjoyable medium of communication and adhesion in our profession.

Without the 'team' we are lost, remembering the adage T.E.A.M., Together Each Achieves More.

I must, however, pick up on something with which I don't agree and hope you will take this in the spirit in which it is delivered.

Here we are in only your second edition saying 'My training and experience is being used to the full here... I am appreciated and respected and because you have so much more responsibility than in the UK, you are valued more'.

We have a shortage of good dental nurses here in the UK. Not only are things changing (albeit slowly) regarding expanding the duties of nurses, but I value my nurses here as much, if not more than those I had practising in Canada, for more than 20 years.

Our patients here have in many instances simply not had the 'choices' of our North American cousins, and I'll be the first to admit that this culture has damaged the public's perception of UK dentistry and, therefore, their expectations. This is the challenge. The challenge is to change all that; and that we can only do as a cohesive team.

We've got boundless beauty here in the British Isles and whilst I saw beauty and majesty in my home in British Columbia, I am proud and determined now I'm home.

There's a story of a man in South Africa who longed to hunt for diamonds and would set off from his farm for weeks on end. He sold his farm eventually to search further afield, only to discover that the new owner had discovered, what turned out to be, a seam of diamonds in the river running through the property. The moral of the story is, don't overlook those things you have now, remember the grass is always greener...

My partner, team and I are working to raise the awareness locally by developing a truly outstanding practice providing the latest technology and greatest care available. We believe we can bloom where we're planted and I believe others can too.

Thank you for an excellent journal.

Stephen J. Bray, Dentist, Dorset

\section{Vital responds}

Hear! Hear! We applaud your comments and totally agree! We shall be taking your point and hope to have a feature shortly based in a beautiful place in the UK.

\section{This is your page to write to and open up topics for debate, reply to each other and offer advice. The letters we have for this issue promote UK dentistry and investment in the term 'PCD'. What are your thoughts and contributions? These can be about your profession only or about working in dentistry in general.}

\section{Military nurses}

I am writing in response to the 'Career pathways' article in the previous issue of Vital, specifically page 25 and the paragraph titled 'Armed Forces'. The article refers to 'dental clerk assistants'. This term is incorrect. Within the Defence Dental Agency, military dental nurses belong to the Royal Navy, Army and Royal Air Force. The correct terminology for military dental nurses is:

Within the Royal Navy they are Dental Surgery Assistants.

Within the Army they are Dental Support Specialists.

Within the Royal Air Force they are Dental Nurses.

\section{Captain Paul Leighton}

Corporate Communications Officer

Headquarters Defence Dental Agency

\section{Vital replies}

We would like to thank Captain Leighton for this clarification and also his contribution to our feature on military dentistry in this issue, pages 27-30.

\section{Correction}

Following correspondence from students on the NVQ Oral Health, Leicester, we wish to apologise for any misunderstanding that may have arisen from a miswording in our Spring Letters page. We reported that '10 students had completed the course in December 2003'. Kath Payne actually wrote that ' 10 students will complete the course in December 2003'. 


\section{'PCD' worth promoting}

Having read the first two issues of Vital I have been delighted to discover a journal of quality that finally recognises Professionals Complementary to Dentistry (PCDs).

However, on reading the letter of one of my colleagues from the National Examining Panel, James H. Hardy, Surrey, I was disappointed by his suggestion that it was time to drop the title PCD. Surely the fact that trainee dental nurses and patients do not understand the term, suggests it's time for a little more education and publicity to raise our profile higher. It took years to change the mindset of some from DSA to dental nurse.

The term PCD is still in its infancy. In the NHS primary care sector a similar acronym is used AHP - Allied Healthcare Professionals, including among others occupational therapists, podiatrists, district nurses and health visitors. This is a very influential professional body. As I work in the primary care sector I was only too pleased when the dental profession finally came up with the title PCD. At last we became a recognisable professional group, one to be given consideration by Primary Care Trusts (PCTs). As an active participant in several PCT committees including Infection Control and Health and Safety, I became aware that many of my fellow healthcare workers previously considered dentistry the sole domain of dentists. We cannot abandon the combination of the various dental professional groups that go to make up PCDs. I believe in time this too will be as strong and influential as the AHPs who retain the individuality of their different professional groups but have additional strength in numbers under the one name.

Vital is one the first outward recognitions for PCDs by the dental profession as a whole and long may it continue, but unfortunately it seemed to remain hidden under the BDJ. I made this observation after visiting several general dental practices during March and April. At each visit I showed the first issue of Vital to all the staff, none of the PCDs had seen it before. All of the dentists had it, but had failed to pass it onto their staff - without any substantial explanation as to why.

Change is a difficult notion for many people and at present dentistry is going through possibly its greatest upheaval with Options for Change, Statutory Registration and Agenda for Change. Not all change is for the best but I feel optimistic for the future and all that it holds for PCDs, especially dental nurses who have waited a very long time and worked hard toward gaining statutory registration, their patience and determination is to be finally rewarded. Hopefully with the opening up of career pathways with the use of their skill-mix and continued professional development, their job satisfaction and recruitment will improve.

\section{Janet Prosser}

PDS Project Manager, Cheshire

\section{SEND YOUR LETTERS to: the Editor, Vital,} Publishing Directorate, British Dental Association, 64 Wimpole Street, London W IE 2UZ or email vital@bda.org. Authors must sign the letter, which may be edited for reasons of space.

\section{Our star letter writer} will receive a Sonnet Black Laque fountain pen (£90 RRP) courtesy of Parker Pens.

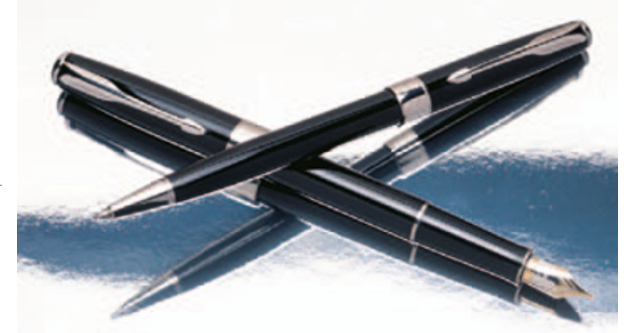

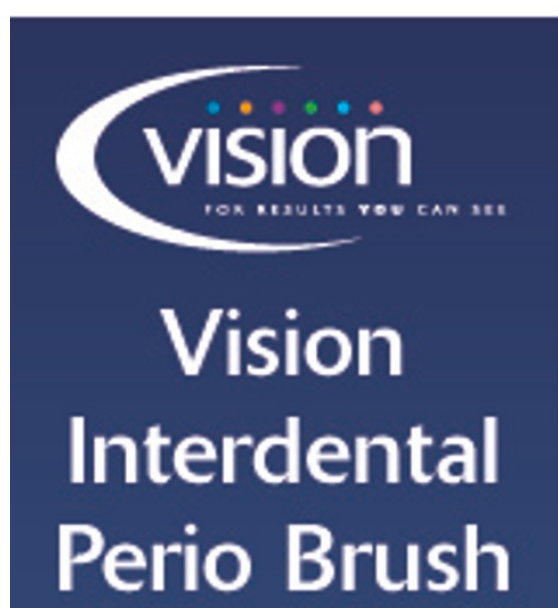
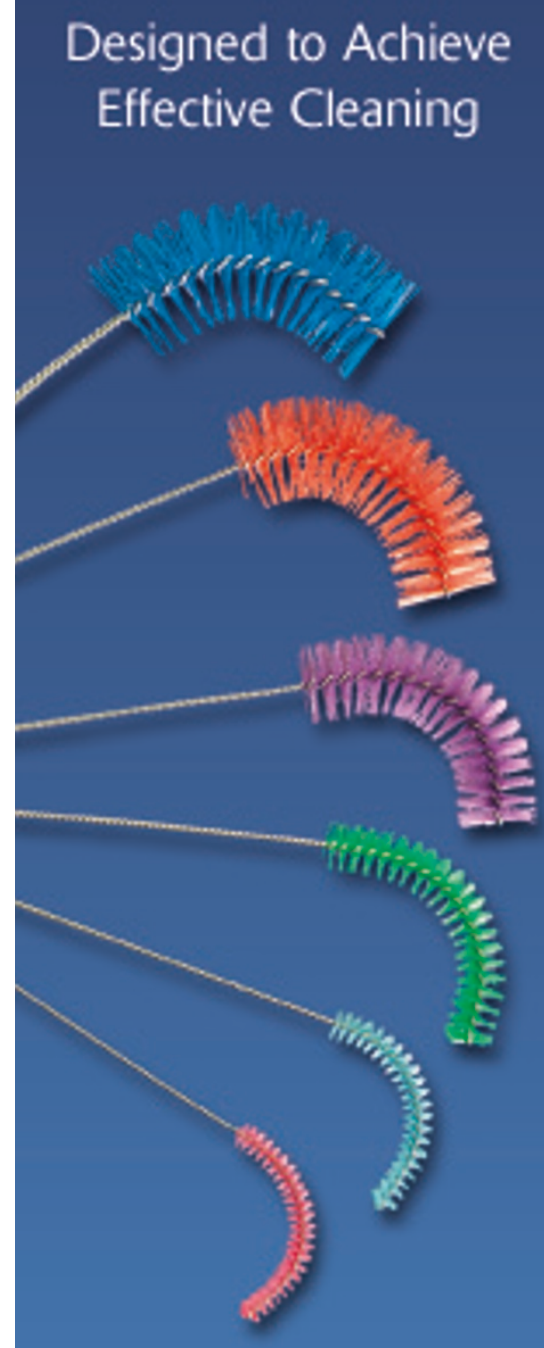

Curved brush head to clean deep into the interdental space

Choice of sizes to achieve a close fit and optimum clean

Coloured filaments to promote use by showing plaque and debris removed

08456345000 www.visionperio.com Call for a free sample 